\title{
Three-loop Corrections to the Mass of the Light Higgs Boson in the MSSM
}

\author{
Philipp KANT* \\ TTP Karlsruhe \\ E-mail: philipp.kant@kit.edu
}

The Minimal Supersymmetric extension of the Standard Model (MSSM) predicts the existence of a light neutral Higgs boson. Once found at the LHC, its mass will immediately become a precision observable. The theoretical value of the Higgs mass $M_{h}$ is subject to large radiative corrections. Due to the large top Yukawa coupling, loops of top quarks and their superpartners provide the dominant contribution to the radiative corrections.

We present a calculation of the SUSY-QCD corrections to these diagrams, up to the three-loop order. We find that our three-loop results can be in the range of one $\mathrm{GeV}$, and are thus relevant when compared with the expected experimental accuracy at the LHC. We also find a significantly reduced dependency on the renormalisation prescription, thus decreasing the theoretical uncertainty of the prediction of $M_{h}$.

European Physical Society Europhysics Conference on High Energy Physics

July 16-22, 2009

Krakow, Poland

${ }^{*}$ Speaker. 


\section{Introduction}

An important feature of the minimal supersymmetric extension of the Standard Model (MSSM) is the existence of a light neutral Higgs boson. The MSSM Higgs sector is a Two-Higgs Doublet Model, the parameters of which are related to the gauge couplings through Supersymmetry. These relations reduce the number of new (in comparison with the Standard Model) parameters to two, which are usually chosen to be the ratio $v_{2} / v_{1}=\tan \beta$ of the vacuum expectation values of the two Higgs doublets and the mass $M_{A}$ of the pseudoscalar Higgs. Once these are fixed, the mass $M_{h}$ of the light neutral Higgs boson is not a free parameter, but a calculable prediction of the theory. At the tree level, there is an upper limit $M_{h} \leq M_{Z}$. At higher orders, this bound gets shifted up by radiative corrections to the Higgs self-energy, which depend on the spectrum of the superpartner masses.

The one- and two-loop corrections to $M_{h}$ have been extensively studied in the literature (for reviews, see e.g. Refs. [1,2]). The studies show that, due to the large top-Yukawa coupling, the most sizeable corrections stem from loops of top quarks and their superpartners in the Higgs propagator. For a light Higgs boson, the Large Hadron Collider (LHC) will be able to measure its mass with an expected experimental accuracy of $100-200 \mathrm{MeV}$ [3]. To take full advantage of the experimental data, the theoretical prediction for $M_{h}$ has to match this precision. However, based mostly on the renormalization scale and scheme dependence, the theoretical uncertainty on the prediction of $M_{h}$ has been estimated to $3-5 \mathrm{GeV}[2,4]$.

While there have been many publications tackling the one- and two-loop corrections to $M_{h}$, there are only two calculations available that go to the third order of perturbation theory. In [5], Renormalisation Group methods have been used to calculate the leading- and next-to leading term in $\ln \left(M_{S U S Y} / M_{t}\right)$, where $M_{S U S Y}$ is the typical scale of SUSY particle masses. Motivated by the fact that the corrections from top and stop loops dominate the overall corrections, the three-loop SUSYQCD corrections to these diagrams have been computed in [6]. Because current methods are not sufficient to solve three-loop multiscale integrals exactly, the calculation in [6] assumed a strong hierarchy among the superparticle masses and performed nested asymptotic expansions [7] to reduce the problem to single-scale integrals. While [6] considered two rather simple mass hierarchies, it is the aim of this talk to report on recent progress on computing more involved scenarios and discuss which are important for phenomenological studies.

\section{Outline of the Calculation}

We calculate the corrections to $M_{h}$ by evaluating virtual corrections to the Higgs propagator. As in [6], we restrict ourselves to diagrams where the Higgs couples to top quarks or their superpartners, including SUSY-QCD corrections up to Order $\mathscr{O}\left(\alpha_{s}^{2}\right)$. This leaves us with the following virtual particles: top quarks $t$ and their superpartners, the stops $\tilde{t}_{1 / 2}$, the gluons $g$ and gluinos $\tilde{g}$, as well as the light quarks $q$ and squarks $\tilde{q}$, which enter at the three-loop level.

One can perform the calculation assuming different hierarchies amongst the superpartner masses. To estimate the error introduced by expanding around these hierarchies, we systematically compare, at the two-loop level, with the exact result which is given in [8] in very compact 

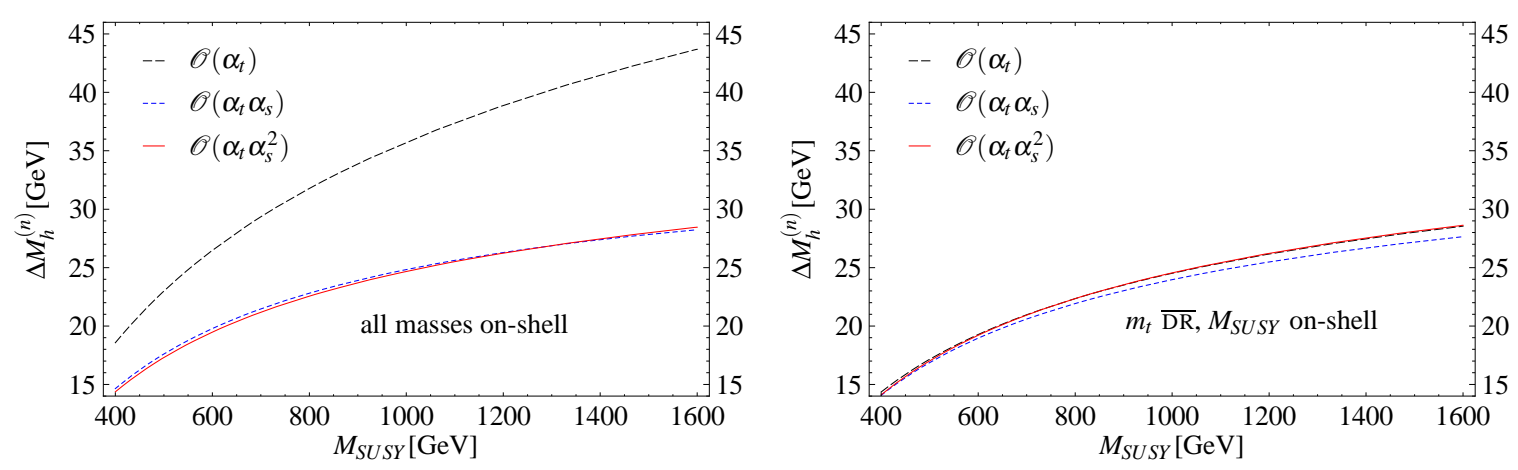

Figure 1: Renormalisation scheme dependence of the corrections $\Delta M_{h}=M_{h}-M_{h}^{\text {tree }}$. This figure assumes a degenerate mass spectrum of the SUSY particles. In the left panel, all masses are renormalised on-shell, while in the right panel the $\overline{\mathrm{DR}}$ scheme is used for the top mass. The masses of the SUSY particles are renormalised on-shell in both cases, to ensure that $\Delta M_{h}$ is plotted over the same parameter. Choosing the $\overline{\mathrm{DR}}$ scheme also for the superpartner masses has a small effect on the Higgs mass.

form. A detailed study of this comparison, which shows that the relative error can be brought below $5 \%$ for the SPS benchmark scenarios from [9], will be presented in a forthcoming publication [10].

\section{Renormalisation Prescription Dependence}

We use Dimensional Reduction [11] in order not to spoil Supersymmetry through the regularisation. This leaves us with the choice between using on-shell renormalisation conditions and using minimal subtraction, i.e. the $\overline{\mathrm{DR}}$ scheme. In order to make a justified choice for one scheme over the other, we analysed the magnitude of the corrections of different loop orders in both schemes (see Fig. 1).

The figure demonstrates three points: $i$ ), the large one-loop corrections to $M_{h}$ are an artefact of using the on-shell top mass. The corrections of higher orders are significantly smaller in the $\overline{\mathrm{DR}}$ scheme, indicating a better behaviour of the perturbative expansion, ii), while there is a significant deviation between the two schemes at the one-and two-loop level, at the third loop order the discrepancy all but vanishes, indicating a stabilisation of the theoretical prediction, and iii), the three-loop corrections amount to some hundred MeV. After this analysis, we resolved to use the $\overline{\mathrm{DR}}$ scheme for the renormalisation of our parameters. An exception to this is the mass of the $\varepsilon$-scalar that appears in Dimensional Reduction, which we renormalise on-shell and set it to zero.

\section{Results}

As an example for the numerical impact of our calculation we show results for the SPS benchmark line SPS2. Fig 2 shows $M_{h}$ including corrections of one-, two- and three-loop order in black, blue and red, respectively. To get the best prediction possible, we extract all available one- and two-loop corrections from the program FeYNHigGS [4, 12-14]. In particular, we use the exact two-loop result from [8] for the $\mathscr{O}\left(\alpha_{t} \alpha_{s}\right)$ corrections. It is notable that the three-loop corrections amount to about one $\mathrm{GeV}$ for large $m_{1 / 2}$. 


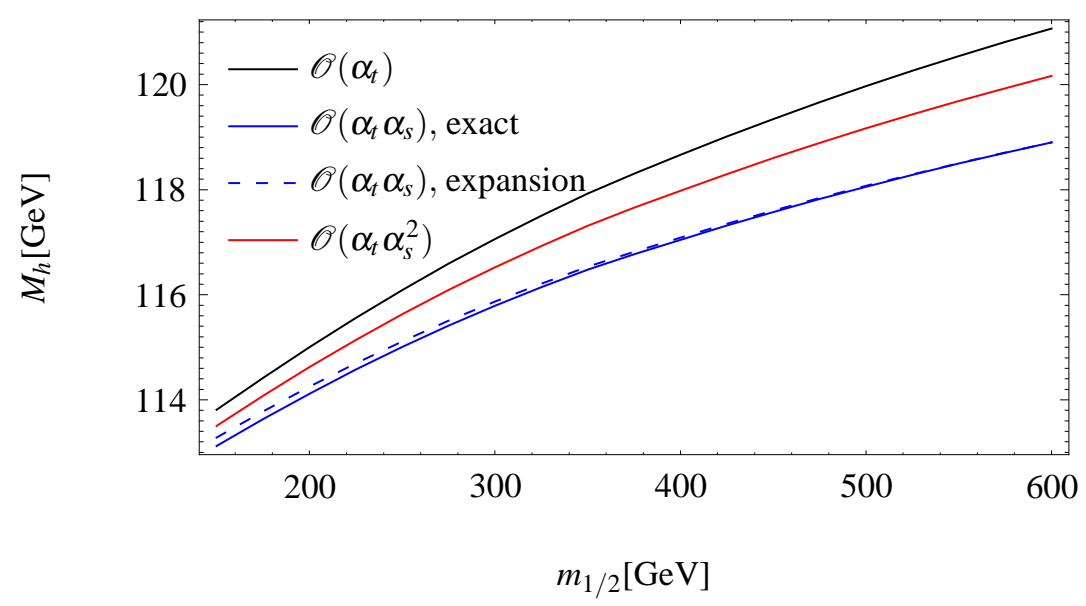

Figure 2: Higgs mass for the benchmark line SPS2. The red line includes our three-loop corrections, as well as all the corrections of one- and two-loop order that are implemented in FeYNHIGGS.

\section{References}

[1] S. Heinemeyer, Int. J. Mod. Phys. A 21 (2006) 2659 [arXiv:hep-ph/0407244].

[2] B. C. Allanach, A. Djouadi, J. L. Kneur, W. Porod and P. Slavich, JHEP 0409 (2004) 044 [arXiv:hep-ph/0406166].

[3] CERN Report No. CERN-LHCC-2006-021; CMS-TDR-008-2, 2006, http://cmsdoc.cern.ch/cms/cpt/tdr/.

[4] G. Degrassi, S. Heinemeyer, W. Hollik, P. Slavich and G. Weiglein, Eur. Phys. J. C 28, 133 (2003) [arXiv:hep-ph/0212020].

[5] S. P. Martin, Phys. Rev. D 75 (2007) 055005 [arXiv:hep-ph/0701051].

[6] R. V. Harlander, P. Kant, L. Mihaila and M. Steinhauser, Phys. Rev. Lett. 100 (2008) 191602 [Phys. Rev. Lett. 101 (2008) 039901] [arXiv:0803.0672 [hep-ph]].

[7] V. A. Smirnov, Springer Tracts Mod. Phys. 177 (2002) 1.

[8] G. Degrassi, P. Slavich and F. Zwirner, Nucl. Phys. B 611 (2001) 403 [arXiv:hep-ph/0105096].

[9] B. C. Allanach et al., in Proc. of the APS/DPF/DPB Summer Study on the Future of Particle Physics (Snowmass 2001) ed. N. Graf, Eur. Phys. J. C 25 (2002) 113 [arXiv:hep-ph/0202233].

[10] R. V. Harlander, P. Kant, L. Mihaila and M. Steinhauser, in preparation

[11] W. Siegel, Phys. Lett. B 84 (1979) 193.

[12] M. Frank, T. Hahn, S. Heinemeyer, W. Hollik, H. Rzehak and G. Weiglein, JHEP 0702 (2007) 047 [arXiv:hep-ph/0611326].

[13] S. Heinemeyer, W. Hollik and G. Weiglein, Eur. Phys. J. C 9 (1999) 343 [arXiv:hep-ph/9812472].

[14] S. Heinemeyer, W. Hollik and G. Weiglein, Comput. Phys. Commun. 124 (2000) 76 [arXiv:hep-ph/9812320]. 